\title{
The Four Cs of HIV Prevention with African Americans: Crisis, Condoms, Culture, and Community
}

\author{
John K. Williams • Gail E. Wyatt • Gina Wingood
}

Published online: 21 August 2010

(C) The Author(s) 2010. This article is published with open access at Springerlink.com

\begin{abstract}
HIV/AIDS continues to be a devastating epidemic with African American communities carrying the brunt of the impact. Despite extensive biobehavioral research, current strategies have not resulted in significantly decreasing HIV/ AIDS cases among African Americans. The next generation of HIV prevention and risk reduction interventions must move beyond basic sex education and condom use and availability. Successful interventions targeting African Americans must optimize strategies that integrate sociocultural factors and address institutional and historical barriers that hinder or support HIV risk reduction behaviors. Community-based participatory research to decrease the HIV/AIDS disparity by building community capacity and infrastructure and advocating for and distributing equitably, power and resources, must be promoted. Recommendations for paradigm shifts in using innovative theories and conceptual frameworks and for training researchers, clinicians, grant and journal reviewers, and community members are made so that culturally congruent
\end{abstract}

\footnotetext{
J. K. Williams $(\bowtie) \cdot$ G. E. Wyatt

Department of Psychiatry and Biobehavioral Sciences,

Semel Institute for Neuroscience and Human Behavior,

University of California, Los Angeles,

760 Westwood Plaza, C8-871C,

Los Angeles, CA 90024-1759, USA

e-mail: Keoniwmd@aol.com

G. E. Wyatt

e-mail: gwyatt@mednet.ucla.edu

G. Wingood

Department of Behavioral Sciences and Health Education,

Rollins School of Public Health, Emory University,

1518 Clifton Road NE,

Atlanta, GA 30322, USA

e-mail: gwingoo@sph.emory.edu
}

interventions may be tested and implemented at the community level.

Keywords HIV prevention - African American · Culture . Condoms

\section{Introduction}

The Context

In the United States, HIV/AIDS continues to be a devastating epidemic within African American communities, posing a significant public health crisis [1]. HIV/AIDS represents a health disparity where blacks comprise $12 \%$ of the population but account for a disproportionately high number of the cases $[1,2]$. The latest Centers for Disease Control and Prevention (CDC) statistics cite $51 \%$ of the 42,655 new HIV/AIDS diagnoses among blacks [1]. Blacks also account for $48 \%$ of the more than half million individuals living with HIV/AIDS [1]. While the public health response over the past nearly three decades has been to develop, test, and disseminate HIV prevention and risk reduction interventions, HIV/AIDS rates continue to be the worst among blacks as compared to other racial/ethnic groups [1]. The CDC and researchers in the public and private domains have sought to identify factors that heighten HIV risks and promote disease infection and transmission [3]. Despite evolving strategies that address the increasingly acquired information about the infection, there has been minimal translation to decreasing HIV/AIDS within African American communities.

When HIV was initially identified, prevention efforts targeted certain risk groups [4, 5]. As the epidemic progressed in the mid-1990s, increasing numbers of 
infected black, non-Hispanic populations surpassed those of whites $[1,6]$. Interventions focused on educating the general population on the modes of HIV/AIDS transmission and attention was placed on barriers to accepting and using condoms and sexual decision-making that heighten HIV infection and transmission risks [7, 8]. Importantly, it became more apparent that in the absence of a biomedical cure for HIV, behavior change was and still is, the most effective strategy in decreasing HIV infection, re-infection, and transmission $[9,10]$. This is especially significant given that sexual behaviors continue to be the most common mode of HIV transmission, and understanding the processes involved in sexual decision-making is necessary to ensure long-term behavior change.

\section{Going Beyond Condoms}

In 1996, the Prevention Research Branch, Division of HIV/ AIDS Prevention (DHAP) at the CDC initiated the HIV/ AIDS Prevention Research Synthesis (PRS) Project, whose purpose was to review and summarize the HIV behavioral prevention research literature, identifying efficacious interventions. The PRS Project created a compendium whereby HIV/AIDS health care providers and community-based organizations (CBOs) could select and implement programs most appropriate for their target populations. While these programs have been evidenced-based, there continues to be barriers to implementing them in the real world, including their uptake by potential recipients who may not be motivated to participate without financial incentives. Financial incentives are commonly used in the testing of HIV interventions, but questions of successful programs arise when incentives are not part of community services. Further, the basic premise of these interventions was that knowledge influences behaviors and that by providing HIV education and access to condoms, HIV infection rates would decrease. Unfortunately, behavior change is complicated and multifaceted, and "cookie-cutter" models have not necessarily been proven to be translatable across diverse populations and attempts to do so have been fraught with challenges [11].

Identification of moderating and mediating variables that influence sexual behaviors and HIV transmission is critical to risk reduction if HIV interventions are to be successful in African American communities. One such variable is culture, which includes customs, lifestyle, activities, values, and beliefs, all of which are pertinent to individual sex roles. However, culture is only one contextual variable that must be included in HIV interventions [12•, 13-16]. The next generation of interventions targeting African Americans need to go beyond basic sex education and condom use and availability, acknowledging limitations of earlier interventions. Condom use must also be examined within a broader sociocultural context. For example, the decision to use condoms is frequently decided upon at the couple's level, which was evident with the Eban intervention for HIV serodiscordant African American couples [17]. Alternatively, population-level indicators of HIV risks such as partner availability and concurrency may also influence condom use [18, 19]. Finally, societallevel interventions, such as reducing HIV stigma, may influence HIV testing and possible condom use. Thus, HIV interventions must address the historical and institutional factors that can affect individual, as well as interpersonal decision-making, integrating the sociocultural context with critical core elements of community relationships and infrastructure. Without acknowledging the context and the strengths and challenges of African Americans within their communities, behavioral models of HIV risk reduction will only be plagued with limitations that do not provide the skills or problem solving strategies to maintain long-term behavioral change. This next generation of interventions must lend attention to core cultural and community elements, which will facilitate individual HIV risk reduction, but also support successful translation and implementation at the couple and community level.

\section{Addressing Culture}

After thoroughly reviewing evidenced-based interventions, Williams et al. [20••] identified six core intervention elements: 1) gender specificity; 2) distinct target population; 3) theoretical foundation; 4) cultural and historical congruence; 5) skill-building components; and 6) welldefined goals. While most successful interventions address four of these core elements, two are commonly neglected. Applying appropriate theoretical models and focusing attention to cultural and historical factors tend to be commonly ignored or only minimally addressed.

\section{Conceptual Framework: Finding Appropriate Theoretical Models}

The first generation of HIV prevention research focused on mainly one prevention strategy, safer sex with condom use. They minimally addressed and included African American history and culture [21], or how these factors could impact individual experiences and sexual decision-making. Prevalent was the notion that the elevated HIV/AIDS rates had to do solely with individual behavior and not the sociocultural context of health disparities, lack of health insurance and employment, and poverty that have long existed. Consequently, research promoted an individualistic approach to risk taking practices. Another limitation was that it assumed homogeneity across groups, which erroneously supported the notion that HIV risk factors were universally the same 
[21]. Subsequently, health promotion interventions developed for whites were extensively applied to those from other racial/ethnic groups [22]. A second generation of research focused on examining differences in race and ethnicity [23]. During this period, public health researchers commonly classified groups based upon their race/ethnicity, believing it was a valid proxy for culture [16]. Attempts by researchers to address and incorporate historical patterns of behavioral change and cultural beliefs and values that contradicted prevention messages often resulted in myopic interpretations and oversimplification of the complexities and depth of differences between ethnic groups and their risk for disease transmission. For example, attention to culture was often represented by racial/ethnic matching of program staff with intervention participants. Also, the inclusion of media resources, such as using videos and music lyrics that included African American models or was written by African American artists, was supposed to demonstrate attention to culture [16]. While ethnic matching may be important [24], the issue of being culturally competent to what is being communicated along with the manner in which the message is conveyed may be as important, if not more important than simply having someone who looks like the intervention participant.

The inclusion and application of culture as a specific social construct in HIV intervention approaches is an innovative concept, which stemmed from the belief that by understanding cultural characteristics of a given group, public health programs would be more capable of meeting the health needs of its members [25]. Unfortunately, only theoretical models that are popular or rooted in a long history of use are commonly used in HIV interventions [12•]. Among models that more specifically address contradictions of some African American cultural values and beliefs is the Sexual Health Model (SHM). Survival coping strategies that transcend risks for HIV/AIDS and need to be incorporated into behavior change efforts have been described [12•]: 1) Adaptive Duality, or saying and doing what is perceived to be acceptable to authority figures while also engaging in behaviors and beliefs that more accurately reflect personal relationships and values; 2) Collectivist Identity that promotes placing others before oneself and perceptions of relationships with others as a sign of personal identity, born out of historical oppression and the unavailability of partners to sustain family life and protection; 3) Indirect Patterns of Communication, born out of necessity and continued as a means of communication to promote social and sexual exchange, but not HIV/ AIDS prevention skills; and 4) Mistrust of Outsiders, a protective mechanism that requires outsiders to earn the trust of African Americans in order to be believable. The SHM addresses these survival patterns and promotes concepts of interconnectedness, body awareness, and sexual ownership to acknowledge historical and contemporary contradictions and reframe these critical strategies.

Lacking cultural elements limits the paradigms that could be developed for African Americans. Obstacles for incorporating culture into interventions include inherent abstractness, a lack of operationalized definitions of how culture can improve an HIV prevention focus, a lack of adequately tested psychometrics to assess culture and its influence on risk behaviors, and the lack of relevant training for those administering the developed interventions (ie, cultural competency) [26•]. Another possible barrier is that reviewers of grants and journal manuscripts may be socialized to expect specific concepts promoted in traditional models. Inclusion of new concepts may create barriers to securing research funding and/or to having research findings published. Concerns about introducing more relevant conceptual frameworks, familiar in ethnic studies or black psychology, may prevent investigators from trying new or different models. Thus, Afrocentric models that motivate ethnic pride and address culturally based mediators of change $\left[16,26^{\circ}\right]$ may not be included in interventions for African Americans.

\section{Cultural Process and Content}

While it may seem intuitive to include and explore how culture influences sexual decision-making by gender, racial/ ethnic group, sexual orientation and/or identification, and sexual-risk and drug-risk behaviors, there is no consensus on how to incorporate it or integrate it as a moderating or mediating variable within HIV prevention models [16]. Two primary strategies have been identified to incorporate culture in HIV prevention interventions [16]. Intervention presentation strategies refer to the communication style and process, with racial/ethnic matching of facilitators or the use of videos with models of the same race/ethnicity, as common examples $[16,22,25,27,28]$. The belief is that there is a decreased chance of miscommunication when information is provided by individuals who look and sound like the participants $[16,22,25,29-31]$. Ethnic minorities must be allowed to define their own language and develop their own dialogue if they are to become empowered in regards to their health [32]. Risk reduction messages must be presented in a manner that speaks to the target population. Further, to ensure the success of the intervention, those who are providing the intervention must be familiar with and ideally be a member of the target population and of the surrounding community.

Curriculum content involves the incorporation of cultural concepts within the intervention. Examples include the discussion of racial or ethnic pride and gender-based power differentials, especially when discussing condom use with sexual partners [29]. Content needs to include not only the 
variables that may directly and obviously affect risk reduction, such as having access to condoms and knowing how to use them, but they must include variables that are culturally rooted. For example, experiences with racism may result in an individual using sex or drugs as a coping strategy and thus, placing themselves at risk for HIV. Other examples may revolve around barriers to seeking health care, expectations regarding African American strength, and mistrust of the health care system. The John Henryism hypothesis, named after the mythic railroad steel driver who overcame challenges only to die from neglecting his limitations, may prevent African Americans from seeking prevention and treatment options [33]. Understanding what guides decisionmaking is influenced by information that is passed from generation to generation about how to sustain life and avoid unexpected outcomes [12•]. Thus, the culture and heritage of an individual must be addressed in order to affect health change.

\section{Other Contextual Variables}

The structure and organization of urban life has largely been segregated from non-ethnic groups, possibly serving as a protective factor for minority populations against stressors such as racism and discrimination. For African Americans, environmental and sociopolitical stressors based upon racial inequities significantly affect their overall health [34]. HIV interventions must address these challenges. In addition to having appropriate conceptual models and including culture, HIV interventions targeting African Americans need to address institutional variables. Institutional variables, rooted in a sociocultural context, may operate on an individual or community and health systems level [35]. At the individual level, structural factors include being poor, lacking health insurance, a regular health care provider and/or access to health care, and having perceptions of discrimination and mistrust of the medical system [35]. At the community and health systems levels, the lack of services or having available only resource-poor cursory or crisis-oriented services, and the lack of culturally competent providers, may all act as barriers to achieving optimal health [35, 36]. Other institutional variables that affect the lives of African Americans and specifically impact sexual decision-making include partnership availability and family dynamics. Social and sexual networks embedded within the complex structure of African American communities have a significant impact on sexual risk behaviors. Limited partner availability from high incarceration rates, poor health and premature death, and high homicide rates may affect sexual relationships and increase risk for HIV through concurrent partnerships rooted in segregated residential neighborhoods, housing complexes, and education and health care systems $[18,19,26 \bullet]$. Further, the meaning of sexual communication and the importance of understanding how a person integrates overt and covert messages about sex and sexuality at the individual level and within the African American family and community have rarely been addressed in HIV interventions [26•].

\section{Cultural Adaptation of Pre-Existing HIV Interventions}

If previously developed interventions are to be implemented, their limitations must first be addressed. Evidence-based HIV interventions (EBI) are now being adapted to enhance their relevancy for numerous at-risk African American populations. Request for applications (RFAs) are being released by the National Institute of Health (NIH) that can bolster the sciencebase of dissemination, adaptation, implementation, and operational research for EBI in the United States [37]. Adaptation kits are being created to enhance the ability of CBOs to adapt evidence-based programs [38]. To reduce racial disparities in HIV, adaptation models such as ADAPTITT [39] can be tailored to address the specific needs of African Americans (Table 1).

\section{Community Endorsement and Support}

Evidenced-based interventions may change risk behavior for study participants, but the truly successful interventions are those that can be implemented in the real world and still produce the same outcomes. Researchers have only recently begun to acknowledge the myriad of challenges in translational implementation research and HIV prevention $[11,39]$. The existing racial disparities in the epidemiology of HIV/AIDS and the extended time that it has taken for research findings to translate into practice have created a national urgency to design effective interventions that can be scaled up rapidly. This context for the translational intervention sciences has produced an important new area of investigation that is now emerging as its own discipline of implementation science [40, 41••]. According to the $\mathrm{CDC}$, implementation research is defined as "the systematic study of how a specific set of activities and designated strategies are used to successfully integrate an evidencebased public health intervention within specific settings" (RFA-CD-07-005). These specific settings, especially as they refer to HIV interventions, are community settings. However, translational research of HIV interventions into African American communities is not without challenges.

The first challenge involves external validity or translating specific findings from evidence-based HIV interventions to real-world situations, HIV/AIDS community agencies, health departments, and sexually transmitted disease (STD) clinics. EBI are usually designed under highly controlled trials, which are typically a very different context of community agencies 
Table 1 ADAPT-ITT questions to ask when adapting HIV interventions

\begin{tabular}{|c|c|c|}
\hline Phase & $\begin{array}{l}\text { Questions to ask when adapting HIV } \\
\text { interventions for populations in general }\end{array}$ & $\begin{array}{l}\text { Questions to ask when adapting HIV interventions for } \\
\text { African Americans }\end{array}$ \\
\hline $\mathrm{A}=$ Assess & What target population is at risk for HIV/AIDS? & $\begin{array}{l}\text { Which particular African American subpopulation } \\
\text { is at risk for HIV/AIDS? }\end{array}$ \\
\hline $\mathrm{D}=$ Decide & $\begin{array}{l}\text { Which evidence-based intervention (EBI) is being } \\
\text { selected for adaptation? }\end{array}$ & $\begin{array}{l}\text { Which EBI is most appropriate to adapt for this } \\
\text { African American subpopulation? }\end{array}$ \\
\hline $\mathrm{A}=\mathrm{Adapt}$ & $\begin{array}{l}\text { How are you documenting the adaptation? } \\
\text { (a) How is the intervention being adapted? } \\
\text { (b) Why is the text being adapted? (c) What } \\
\text { is being adapted? (d) Where did most of the } \\
\text { modifications occur? and (e) What is the } \\
\text { extent of the modification (ie, all sections of } \\
\text { the intervention)? }\end{array}$ & $\begin{array}{l}\text { How are you documenting the adaptation? (a) How } \\
\text { is the intervention being adapted (ie, are culturally } \\
\text { relevant themes being introduced?)? (b) Why is the } \\
\text { text being adapted (ie, is sexual behavior being } \\
\text { contextualized?)? (c) What is being adapted (ie, do } \\
\text { materials need to be adapted so they are culturally } \\
\text { appropriate?)? (d) Where did most of the } \\
\text { modifications occur (ie, culturally tailoring vignettes, } \\
\text { discussion questions, data)? and (e) What is the } \\
\text { extent of the modifications (ie, text and materials } \\
\text { need to be tailored)? }\end{array}$ \\
\hline $\mathrm{P}=$ Production & $\begin{array}{l}\text { Are you producing drafts of the intervention that } \\
\text { reflect changes in the: (a) Core elements? } \\
\text { (b) Logic model? (c) Theoretical framework? or } \\
\text { (d) Study mediators facilitating behavior change? }\end{array}$ & $\begin{array}{l}\text { Are you producing drafts of the intervention that reflect } \\
\text { changes in the: (a) Core elements (ie, to be more } \\
\text { culturally congruent)? (b) Logic model (ie, to } \\
\text { acknowledge culture)? (c) Theoretical frameworks (ie, } \\
\text { to include culturally appropriate models [ie, CBPR, } \\
\text { empowerment theory])? or (d) Mediators addressing } \\
\text { culturally appropriate mediators (ie, African American } \\
\text { community involvement)? }\end{array}$ \\
\hline $\mathrm{T}=$ Topic Experts & $\begin{array}{l}\text { Are topic experts from the university involved in } \\
\text { adapting the intervention? }\end{array}$ & $\begin{array}{l}\text { Are topic experts from the African American } \\
\text { community involved in adapting the intervention? }\end{array}$ \\
\hline $\mathrm{I}=$ Integrate & Is text being integrated from the target population? & $\begin{array}{l}\text { Is text being integrated that is appropriate for the } \\
\text { subpopulation's reading level? }\end{array}$ \\
\hline $\mathrm{T}=$ Train & $\begin{array}{l}\text { Can facilitators implement the adapted intervention } \\
\text { with fidelity? }\end{array}$ & Are facilitators culturally competent? \\
\hline $\mathrm{T}=\mathrm{Test}$ & $\begin{array}{l}\text { Is the intervention feasible, efficacious, and cost } \\
\text { effective? }\end{array}$ & $\begin{array}{l}\text { Is the intervention acceptable to the African American } \\
\text { subpopulation? }\end{array}$ \\
\hline
\end{tabular}

CBPR — community-based participatory research

that vary in culture, fiscal, and agency resources, and organizational capacity [42]. This "top-to-bottom" approach to implementation with researchers instructing community agencies is a well-entrenched method of instituting behavior change among high-risk populations, which has limitations. While it is an almost implicit assumption that EBI will have to be adapted for real-world settings, researchers are challenged in making this translation. As a solution, community-based participatory research (CBPR) has been proposed. As defined by the Agency for Healthcare Research and Quality in 2004, CBPR is "a collaborative research approach that is designed to ensure and establish structures for participation by communities affected by the issue being studied, representatives of organizations, and researchers in all aspects of the research process to improve health and well-being through taking action, including social change" [43]. The strength of CBPR is that it respectfully integrates culturally and practice-based evidence and indigenous research methodologies. However, many of the practices and programs promoted by communities have not been formally evaluated. Also, CBOs may not possess the scientific knowledge and skills to adapt supported interventions [42, 44].

A second challenge to translational research is obtaining community "buy-in" to programs that may not include culturally congruent beliefs, practices, and theories. CBOs have significant access to and rapport with the target population and without their support, interventions may not be accepted, endorsed, or reach the community in the form that they were initially developed. In order to develop a mutually respectful and equitable relationship with community agencies, community partners must be included at all stages of the intervention development process and not only in the translational stage $[45 \bullet \cdot, 46]$. Recognition of the diverse community partners must be acknowledged and not simply limited to HIV/AIDS service providers. African American churches and other spiritual and civic organizations are often asked to endorse community health 
promotion programs. Their inclusion in developing and adapting interventions is critical.

A third challenge is the actual implementation of evidence-based HIV interventions. While university-based researchers typically develop the interventions, they are either not involved or minimally involved in the actual packaging, manualizing, and dissemination of these interventions. Manualizing is a lengthy process and not often rewarded in peer-reviewed publications or other products deemed valuable to university faculty. Additionally, the development of core elements, logic models demonstrating how the intervention operates, and project impact measures is commonly conducted with limited input from university faculty. Exacerbating this issue is the fact that implementation science is so new that many university faculty are not familiar with the terms commonly used in translational research. Thus, the original researchers typically have not been given due consideration for what constitutes core elements or a logic model, or what appropriate project impact measures would be for their evidence-based HIV intervention.

Finally, the fourth challenge is addressing sustainability of an HIV intervention. Approaches such as CBPR may be helpful in establishing and formalizing long-term relationships to facilitate sustainability. Investing in community and grassroots organizations by providing resources and trainings, acknowledging diversity and strengths of minority agencies, and endorsing culturally congruent interventions may facilitate the success of intervention implementation.

\section{Conclusions}

To best combat the HIV/AIDS epidemic within African American communities, researchers, clinicians, and community partners must collaborate to develop culturally congruent HIV interventions that go beyond traditional strategies. While sex education and condom promotion are both critical and necessary weapons, only by understanding the importance of the historical and sociopolitical context of an individual's life, the influence of culture and the normative scripts that may contradict cultural values and interfere with prevention messages, and the significant contribution of community partners, will this epidemic be curbed. Emphasis on improving the infrastructure of CBOs so that they have resources to continue interventions once research studies are completed is necessary. To best address HIV/AIDS in African American communities, the following suggestions are recommended:

1. Researchers need to endorse theories and conceptual frameworks that are pertinent to African Americans and their sociocultural context. Past interventions have failed to focus on culture and have stressed individual risk behaviors, the divergence from social norms, and sexual behaviors within a non-contextual setting. Innovative models must develop culturally congruent prevention approaches that place emphasis on structural and social contextual factors [12•, 47], including:

a. family, partnerships, community, and the collective nature of African Americans;

b. traditional norms imposed by individuals or community organizations such as churches and faith agencies, which may not promote positive personal control (ie, the individual deciding what is best for them);

c. cultural pride, creativity, and imagination and victory in overcoming challenges; and

d. sexual behaviors that may stem from hostile historical and contextual situations such as settings of poverty and abuses that occur between partners (sexual, physical abuse), and within communities (neighborhood violence), institutions (incarceration in the correctional system), and society (gender stereotypes promoted by media).

These theories must also examine the confluence of personal, environmental, and institutional factors over time, as they may discourage the maintenance of improved health.

2. Studies must draw upon the strengths and resiliency of African American communities and not focus solely on deficit models. Thus, case controlled studies of individuals at risk compared to those who are not at risk are needed to better understand how individuals with similar demographic characteristics avoid HIV infection and/or cope with health challenges such as histories of trauma and mental health disorders.

3. Emphasis must be placed on HIV risk reduction as well as prevention messages. Prevention strategies targeting HIV-negative African Americans tend to overshadow the needs of reducing HIV re-infection among those who are already infected or transmission to their partners. Condoms, when presented within a sociocultural context, can be a powerful weapon against HIV even for those already infected. For example, a recent study with 535 HIV serodiscordant African American couples highlighted significant change in the number of incidents of unprotected sex and proportion of condom use in a risk reduction intervention compared to a health promotion control [17]. This study stressed the importance for those who are living with the virus to be able to maximize intimacy within their relationships and reduce HIV-related risks with their HIV seronegative partners. 
4. Multidisciplinary collaborations to holistically treat individuals are vital for the success of HIV interventions. Sexual health must be integrated along with physical and psychological health. Importantly, HIV/ AIDS researchers need to integrate black psychology in order to identify new concepts that have been overlooked and understudied, which may impact sexual decision-making and motivations for improving overall health.

5. Interventions need to be sensitive to perceived racial/ ethnic, gender, and sexual orientation stigma and address how multiple minority statuses for African Americans can weigh on individuals and affect their lives. Also, since these mediators are rarely included in research, investigators must endorse and advocate for new measures to assess these variables.

6. Finally, researchers, clinicians, and community partners all need to be culturally competent in order to work with at-risk African Americans. Thus, cultural competency trainings must be developed. It is also important to address the dearth of African Americans who are funded to conduct research with African Americans [48]. While there are some efforts to increase the number of racial and ethnic minority investigators [49], currently, very few receive adequate research funding [50]. In addition to needing training and mentoring programs to conduct culturally congruent research, it is necessary to foster an open dialogue with established reviewers of grants and journals on the introduction of nontraditional and innovative concepts that need further study.

In summary, developing HIV interventions that only replicate what earlier interventions accomplished will not decrease the prevalence of HIV/AIDS in African American communities. Acknowledgment of the limitations that currently exist in HIV research is necessary so that innovative translational biomedical research can advance. Research strategies and community approaches that are consistent with African American ideology and culture and that address the challenges of African Americans have the potential for instituting successful behavior change.

\begin{abstract}
Acknowledgments The authors would like to express their appreciation to Tony Earls, MD, HSPH, for his initiative in bringing together the authors and for his support of this manuscript. Funding to write this manuscript was provided from the National Institute of Mental Health (Center for Culture, Trauma, and Mental Health Disparities [5P50MH073453] and the ES-HIM Project [1 R34 MH077550]), the National Center on Minority Health and Health Disparities (UCLA-Drew Project EXPORT-2P20MD000182), and the UCLA Center for AIDS Research (CFAR) NIH/NIAID AI028697 and the UCLA AIDS Institute.
\end{abstract}

Disclosure No potential conflicts of interest relevant to this article were reported.
Open Access This article is distributed under the terms of the Creative Commons Attribution Noncommercial License which permits any noncommercial use, distribution, and reproduction in any medium, provided the original author(s) and source are credited.

\section{References}

Papers of particular interest, published recently, have been highlighted as:

- Of importance

- Of major importance

1. Centers for Disease Control and Prevention: Updates to racial/ ethnic disparities in diagnosis of HIV/AIDS - 33 states, 20012005. MMWR 2007, 56:189-193.

2. U.S. Census Bureau: Quick facts, 2000. Available at http:// quickfacts.census.gov/qfd/states/00000.html. Accessed May 2010.

3. Centers for Disease Control and Prevention: Compendium of HIV prevention interventions with evidence of effectiveness. US Department of Health and Human Services 2001. Available at http://www. cdc.gov/hiv/topics/research/prs/index.htm. Accessed May 2010.

4. Coates TJ, Stall RD, Catania JA, Kegeles SM: Behavioral factors in the spread of HIV infection. AIDS 1988, 2(Suppl 1):239-246.

5. Des Jarlais DC, Friedman SR: HIV infection among intravenous drug users: epidemiology and risk reductions. AIDS 1987, 1:67-76.

6. Centers for Disease Control and Prevention: HIV/AIDS surveillance report, 2006. US Department of Health and Human Services 2008, 18.

7. Calsyn DA, Meinecke C, Saxon AJ, Stanton V: Risk reduction in sexual behavior: a condom giveaway program in a drug abuse treatment clinic. Am J Public Health 1992, 82:1536-1538.

8. Prochaska JO: What causes people to change from unhealthy to health enhancing behavior? Cancer Prevention 1990, 1:38-42.

9. Pequegnat W, Stover E: Behavioral prevention is today's AIDS vaccine! AIDS 2000, 14:51-57.

10. Darbes L, Crepaz N, Lyles C, et al.: The efficacy of behavioral interventions in reducing HIV risk behaviors and incident sexually transmitted diseases in heterosexual African Americans. AIDS 2008, 22:1177-1194.

11. Veniegas R, Kao UY, Rosales R: Adapting HIV prevention evidence-based interventions in practice settings: an interview study. Implement Sci 2009, 4:76-84.

12. - Wyatt GE: Enhancing cultural and contextual intervention strategies to reduce HIV/AIDS among African Americans. Am J Public Health 2009, 99:1941-1945. This article describes four protective strategies that may be useful for African Americans and describes the Sexual Health Model. The Sexual Health Model is a culturally congruent model that incorporates established adaptive coping strategies. Importantly, it promotes sexual connectedness, sexual ownership, and body awareness within an African American sociocultural context.

13. Airhihenbuwa C, DiClemente R, Wingood G, Lowe A: HIV/ AIDS education and prevention among African Americans: a focus on culture. AIDS Educ Prev 1992, 4:267-276.

14. Wingood G, Hunter-Gamble D, DiClemente R: A pilot study of sexual communication and negotiation among young African American women: implications for HIV prevention. J Black Psychol 1993, 19:190-203.

15. Wyatt GE: The sociocultural relevance of sex research: challenges for the 1990's and beyond. Am Psychol 1994, 49:748-754. 
16. Wilson BDM, Miller RL: Examining strategies for culturally grounded HIV prevention: a review. AIDS Educ Prev 2003, 15:184-202.

17. El-Bassel N, Jemmott JB, Wingood GM, et al.: NIMH multisite HIV/STD prevention trial for African American couples group: a cluster randomized trial. Arch Intern Med 2010, In press.

18. Adimora AA, Schoenbach VJ, Martinson FEA, et al.: Heterosexually transmitted HIV infection among African Americans in North Carolina. J Acquired Immune Defic Syndr 2006, 41:616-623.

19. Adimora AA, Schoenbach VJ, Doherty I: Concurrent sexual partnerships among men in the United States. Am J Public Health 2007, 97:2230-2237.

20. •• Williams JK, Harawa NT, Ramamurthi HC, Manago C: Learning from successful interventions: a culturally congruent HIV riskreduction intervention for African American men who have sex with men and women. Am J Public Health 2009, 99:1008-1012. This article discusses the importance of addressing historical, structural or institutional, and sociocultural factors that hinder or support HIV risk reduction in high-risk groups, such as African American men who have sex with men. It identifies key elements from successful interventions and discusses how they were incorporated into a community-based HIV risk reduction intervention for African American men who have sex with men and women.

21. Marín G, Burhansstipanov L, Connell CM, et al.: A research agenda for health education among underserved populations. Health Educ Q 1995, 22:346-363.

22. Kreuter MW, Lukwago SN, Bucholtz RD, et al.: Achieving cultural appropriateness in health promotion programs: targeted and tailored approaches. Health Educ Behav 2003, 30:133-146.

23. Pasick RJ: Socioeconomic and cultural factors in the development and use of theory. In Health Behavior and Health Education: Theory, Research, and Practice. Edited by Glanz K, Lewis FM, Rimer BK. San Francisco, CA: Jossey-Bass Inc.; 1997:425-440.

24. Johnson RL, Roter D, Powe NR, Cooper LA: Patient race/ ethnicity and quality of patient-physician communication during medical visits. Am J Public Health 2004, 94:2084-2090.

25. Kreuter MW, McClure S: The role of culture in health communication. Ann Rev Public Health 2004, 25:439-455.

26. - Wyatt GE, Williams JK, Meyers HF: African American sexuality and HIV/AIDS: recommendations for future research. J Natl Med Assoc 2008, 100:44-51. This article discusses the importance of understanding African American sexuality within a culturally congruent and ethnocentric approach to facilitate the decrease of HIV infection and transmission rates among African Americans. It focuses on two major factors, confusion about race-based stereotypes and historical health disparities and mistrust, which have influenced the understanding of African American sexuality. Importantly, it discusses the limitations of what is known and makes recommendations for HIV/AIDS and sexuality research.

27. Airhihenbuwa CO: Health promotion and disease prevention strategies for African Americans: a conceptual model. In Health Issues in the Black Community. Edited by Braithwaite RL, Taylor SE. San Francisco, CA: Jossey-Bass; 1992:267-280.

28. Peterson JL, Bakeman R: AIDS and IV drug use among ethnic minorities. J Drug Issues 1989, 19:27-37.

29. DiClemente RJ, Wingood GM: Randomized controlled trial of an HIV sexual risk-reduction intervention for young AfricanAmerican women. JAMA 1995, 274:1271-1276.

30. Herek G, Gillis JR, Glunt EK, et al.: Culturally sensitive AIDS educational videos for African American audiences: effects of source, message, receiver, and context. Am J Community Psychol 1998, 26:705-744.

31. Wyatt GE, Guthrie D, Notgrass CM: Differential effects of women's child sexual abuse and subsequent sexual revictimization. J Consult Clin Psychol 1992, 60:167-173.

32. Freire P: Pedagogy of the Oppressed. New York, NY: Continuum; 1983.

33. James SA, Hartnett SA, Kalsbeek WD: John Henryism and blood pressure differences among black men. J Behav Med 1983, 6:259-278.

34. Geronimus AT, Hicken M, Keene D, Bound J: "Weathering" and age patterns of allostatic load scores among Blacks and Whites in the United States. Am J Public Health 2006, 96:826-833.

35. Parrish DD, Kent CK: Access to care issues for African American communities: implications for STD disparities. Sex Transm Dis 2008, 35:S19-S22.

36. Heslin KC, Andersen RM, Ettner SL, Cunningham WE: Racial and ethnic disparities in access to physicians with HIV-related expertise: findings from a nationally representative study. J Gen Intern Med 2005, 20:283-289.

37. National Institutes of Health: Dissemination, implementation, and operational research for HIV prevention interventions (R01). PA08-166. Available at http:/grants.nih.gov/grats/guide/pa-files/PA08-166.html. Accessed May 2010.

38. Taylor J, Moore C, Lezin N, et al.: Making Proud Choices Adaptation Kit. Santa Cruz, CA: ETR Associates; 2010.

39. Wingood GM, DiClemente RJ: The ADAPT-ITT model: a model for adapting evidence-based HIV interventions. J Acquir Immune Defic Syndr 2008, 47(Suppl):S40-S46.

40. Greenhalgh T, Robert G, MacFarlane F, et al.: Diffusion of innovations in service organizations: systematic review and recommendations. Milbank Q 2004, 82:581-629.

41. • Proctor EK, Landsverk J, Aarons G, et al.: Implementation research in mental health services: an emerging science with conceptual, methodological, and training challenges. Adm Policy Ment Health 2009, 36:24-34. This article provides an overview for the newly emerging field of implementation science, which will be critical for translating HIV interventions into communities. Key terms and language are defined, conceptualizations and models are explained, and the implications for reseach and training are discussed.

42. Gandelman A, DeSantis L, Rietmeijer C: Assessing community needs and agency capacity - an integral part of implementing effective evidence-based interventions. AIDS Educ Prev 2006, 18(Suppl A):32-43.

43. Viswanathan M, Ammerman A, Eng E, et al.: Communitybased Participatory Research: Assessing the Evidence. Evidence Report/Technology Assessment No. 99 (Prepared by RTI-University of North Carolina Evidence-based Practice Center under Contract No. 290-02-0016). AHRQ Publication No. 04-E022. Rockville, MD: Agency for Healthcare Research and Quality; August 2004.

44. Lehman WE, Greener JM, Simpson DD: Assessing organizational readiness for change. J Subst Abuse Treat 2002, 22:197209.

45. •• Rosenthal MS, Lucas GI, Tinney B, et al.: Teaching communitybased participatory research principles to physicians enrolled in a health services research fellowship. Acad Med 2009, 84:478-484. This article stresses the importance of training and mentoring researchers in CBPR. CBPR proposes to include community partners in all aspects of research in an effort to decrease health 
disparities. Challenges to integrating CBPR to traditional research models are identified and discussed.

46. Jones L, Wells K: Strategies for academic and clinician engagement in community-participatory partnered research. JAMA 2007, 297:407-410.

47. Friedman SR, Cooper HLF, Osborne AH: Structural and social contexts of HIV risk among African Americans. Am J Public Health 2009, 99:1002-1008.

48. Wyatt GE, Williams JK, Henderson T, Sumner L: On the outside looking in: promoting U.S. African American investigator-initiated HIV/AIDS research. Am J Public Health 2009, 99(S1):S48-S53.

49. Forsyth AD, Stoff DM: Key issues in mentoring in HIV prevention and mental health for new investigators from underrepresented racial/ ethnic groups. Am J Public Health 2009, 99(S1):S87-S91.

50. National Advisory Mental Health Council Workgroup on Racial/ Ethnic Diversity in Research Training and Health Disparities Research: An investment in America's future: racial/ethnic diversity in mental health research careers. Rockville, MD: National Institute of Mental Health; 2001. 